\title{
Lupus Glomerulonephritis
}

National Cancer Institute

\section{Source}

National Cancer Institute. Lupus Glomerulonephritis. NCI Thesaurus. Code C34789.

Glomerulonephritis in the context of systemic lupus erythematosus. 